\title{
Nonlinear dynamics of electron flows with density gradient in spherical diodes
}

\author{
M. Virgínia Alves \\ Instituto Nacional de Pesquisas Espaciais, P.O. Box 515, Sao José dos Campos 12201-970, Brazil
}

Graciela Gnavi, Fausto T. Gratton, Sergio Ferro, and César H. Moreno

Instituto de Física del Plasma, CONICET, Facultad de Ciencias Exactas y Naturales,

Universidad de Buenos Aires, Ciudad Universitaria, Pabellón 1, 1428 Buenos Aires, Argentina

(Received 26 October 1999; accepted 17 March 2000)

\begin{abstract}
A variety of Pierce diodes formed by a pair of grounded concentric spheres with electrons streaming radially are investigated with particle-in-cell simulations and analytical models. The electrons flow parallel or antiparallel to a density gradient in convergent or divergent diodes, respectively. The existence and stability (or instability) of laminar steady states with space charge and nonuniform velocity that are possible for a given set of diode parameters, are crucial to determine the outcome of the numerical experiments. The dispersion relation for the stability of the initial constant velocity states and the concomitant energy flow balance are examined in relation to the nonlinear dynamical evolution. Spherical diodes have properties that are common to a dual pair of diodes with reciprocal ratios of collector to emitter radii. Stable oscillatory electrostatic states, associated with Hopf bifurcations, are observed in the simulations. The breakdown of the laminar electron flow has completely different physical characteristics in divergent and convergent diodes. (C) 2000 American Institute of Physics. [S1070-664X(00)01007-7]
\end{abstract}

\section{INTRODUCTION}

The "classical Pierce diode" is a plane diode in which a collisionless cold electron beam propagates between an emitter (located at $x=0$ ) and a collector (at $x=L$ ). Both electrodes are kept at the same potential, which is equivalent to an external short circuit. The electrons leave the emitter with fixed density $n_{0}$ and velocity $v_{b}$. The behavior of the planar Pierce diode is basically determined by the dimensionless Pierce parameter $\alpha=\omega_{p} L / v_{b}\left[\omega_{p}=\left(e^{2} n_{0} / \epsilon_{0} m\right)^{1 / 2}\right.$ : plasma frequency]. The limit current density for the instability onset is obtained from $\alpha=\pi$. Systems with $\alpha>\pi$ are unstable.

Pierce-type instabilities have been invoked in the context of various practical applications and devices such as electron-beam generation, microwave generation and amplification, thermionic converters, drift tubes, triode lasers, and ion-beam neutralization for inertial confinement fusion. A survey of the research on planar Pierce diodes can be found in Ref. 1. The classical Pierce diode model has been extended in many aspects and the literature on the subject is vast. Recently, the Pierce diode was considered as a suitable model for the linear stability of thermionic gas discharges operated at low pressure. ${ }^{2}$ The inclusion of warm plasma effects, ${ }^{3}$ as well as the inclusion of the ion dynamics, ${ }^{4}$ has also attracted the attention of researchers. However, topics like nonlinear steady-state oscillations and chaotic behavior of the system have been developed only in relatively recent times. ${ }^{3,5-9}$

In many electronic devices, thermionic cathodes with large curvature are used for practical reasons. ${ }^{10}$ In fact, electronic devices with cylindrical and spherical electrodes were called "Pierce electrodes" (see e.g., Ref. 11). Experiments with injection of convergent ion beams neutralized by ac- companying electron currents are considered in inertial fusion systems with cylindrical and spherical geometries. In these configurations the Pierce instability effect is relevant. Similarly, the dynamics of the Pierce process including boundaries with curvature is of interest in ion deposition experiments. ${ }^{12-15}$ Divergent beams between spherical electrodes may have a potential for microwave amplification. ${ }^{16}$

The knowledge of the Pierce instability for diodes with curvature is not so well advanced as in the planar case. The linear stability theory for cylindrical and spherical diodes was given in Ref. 16. The nonlinear dynamics of cylindrical Pierce-type devices has been recently investigated using particle-in-cell simulations. ${ }^{17}$ In this paper we present a study of the nonlinear evolution of spherical Pierce-type electrodes, maintained at the same electric potential, using numerical simulations and analytical models. These systems have radial density gradients parallel or antiparallel to the electron flow, depending on whether the emitter focalizes the beam on a collector of smaller radius, or the emitter sends the beam outwards to a collector of larger radius. We will focus our attention on radial perturbations of the steady states with constant or with nonuniform velocity. Angle dependent perturbations will not be considered in this study. As the numerical experiments show, the instability of the constant velocity radial flow does not necessarily imply the end of laminar motion in the diode and the development of a turbulent phase. In fact, the existence and the stability (or instability) with respect to radial perturbations of laminar steady states with space charge (and nonuniform velocity) that are possible for a given set of diode parameters accord- 
ing to the theory, are crucial to determine the final outcome of the numerical experiments. As we shall see, after the linear instability phase the diode may relax directly to a different steady state, characterized by an electrostatic field and a nonuniform velocity flow, without loss of laminarity during this evolution. On the other hand, it may also happen that after a laminar flow disruption the system passes through a transient disordered stage in the electron phase space, which is gradually superseded by a relaxation towards a stable laminar electrostatic state, where order in phase space is recovered.

The research has revealed that some properties, like the states with electrostatic structures towards which the spherical diodes may relax and the frequency of small oscillations about these states, are shared by convergent and divergent diodes with reciprocal values $x_{i}=R_{i} / R_{e}$ and $x_{i}^{\prime}=1 / x_{i}$ of the ratio of the collector radius, $R_{i}$, to the emitter radius, $R_{e}$. This dual characteristic is not present in cylindrical diodes. However, when the initial flow is disrupted and becomes turbulent the nonlinear dynamics revealed by the numerical simulations is quite different in divergent or convergent devices. The investigation has also confirmed that spherical systems are singularized by being stable in the constant velocity configuration for all values of the interelectrode distance $\left|R_{e}-R_{i}\right|$ when the plasma frequency at the emitter is less than $v_{b} / 2 R_{e}$. Planar and cylindrical diodes, instead, become unstable by increasing the interelectrode separation at any injection density.

The layout of the paper is as follows. The geometry of the system is presented in Sec. II. The theoretical concepts that are necessary to interpret the results of the numerical experiments are given in Sec. III, which is subdivided into four parts. In this section we introduce the equations for the fluid model and we examine the existence of alternative laminar electrostatic structures and their stability properties as a function of the parameters of the diode. A description of the instability of the initial constant velocity states, and the connection of the growth (or damping) rate of the perturbative modes with the net energy outflow from the system is also given there. The particle-in-cell model is described in Sec. IV. The results of numerical simulations for divergent and convergent cases, showing the evolution toward stable electrostatic states and other features worthy of notice, are given in the first two parts of Sec. V. This section also contains a third part, where physical differences between divergent and convergent diodes during the breakdown of the laminar flow and the following turbulent stage are highlighted. Finally, our conclusions are presented in Sec. VI.

\section{CONFIGURATION OF THE SYSTEM}

We consider systems with radial flow through, grounded, concentric spherical grids, with radii $R_{e}, R_{i}$, for the emitter and collector electrode, respectively. In the unperturbed steady state, the flow of electrons has a constant radial velocity $v=v_{0}= \pm v_{b}$. We assume that ions are also injected into the system with the same speed and are comoving with the electrons, so that charge and current neutrality are both ensured. This basic setup does not show relative
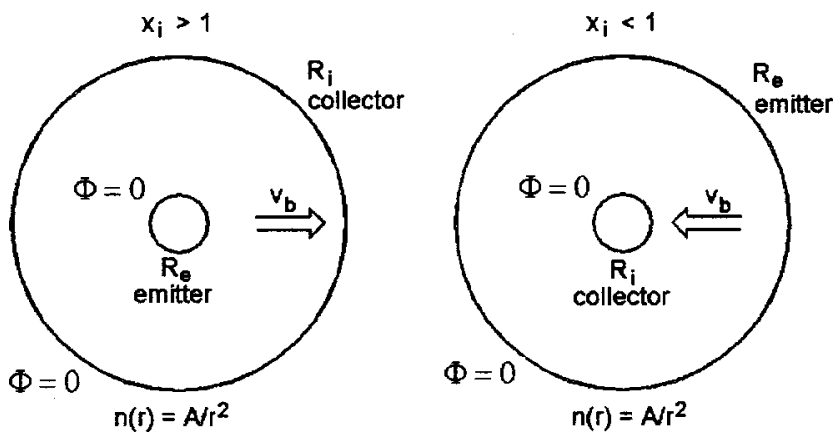

FIG. 1. Set up and geometry of the systems studied in this paper.

electron-to-ion drifts and avoids the possibility of electronion stream instabilities. In this paper we focus the attention on the electrons in view of the high frequency of their dynamics, and we shall ignore the perturbations of the ions (infinite mass). Thus, under the same restriction this study may also apply to a case in which the ions are assumed to be fixed, provided that the ion density is radially varying to achieve charge neutrality. In the comoving setup, for geometrical reasons and because of particle flux conservation under steady state conditions, both electrons and ions have an unperturbed density distribution with a density gradient, $n_{0}=A / r^{2}$, where $A$ is a constant (computed with emitter values, $A=n_{e} R_{e}^{2}$, for instance) and $r$ is the radial position. Thus, initially no electric fields exist in the interelectrode space. Figure 1 shows the two basic configurations studied, with incoming $\left(-v_{b}\right)$ or outgoing $\left(+v_{b}\right)$ radial velocity $v_{0}$, hence forming convergent or divergent flows. In the divergent configuration the electrons flow against the density gradient, conversely in the convergent setup the motion is parallel to the density gradient. This study will bring into light the influence of the convergence or divergence of the electron flow with the concomitant parallel or antiparallel density gradient, on the linear and nonlinear dynamics of the instability.

\section{THEORY}

Previous to a description of the simulation results, we derive in this section several theoretical concepts based on a laminar fluid description, which are necessary to conduct and interpret the numerical experiments properly.

\section{A. The fluid model}

The laminar behavior of the system can be treated using the following model for a monoenergetic beam (cold fluid, pressureless):

$$
\begin{aligned}
\left(\frac{\partial}{\partial t}+v \frac{\partial}{\partial r}\right) v & =\frac{e}{m} \frac{\partial \phi}{\partial r}, \quad \frac{\partial\left(r^{2} n\right)}{\partial t}+\frac{\partial\left(r^{2} n v\right)}{\partial r}=0, \\
\frac{\partial}{\partial r}\left(r^{2} \frac{\partial \phi}{\partial r}\right) & =-\frac{e}{\epsilon_{0}}\left(A-r^{2} n_{e}\right),
\end{aligned}
$$

where $n$ denotes the number density, $v$ the radial velocity of the beam, and $\phi$ the electric potential. In the Pierce diode the boundaries are equipotentials, so that $\phi\left(R_{e}\right)=\phi\left(R_{i}\right)=0$. The remaining boundary conditions are $v\left(R_{e}\right)= \pm v_{b}, n_{e}$ 
$=A / R_{e}^{2}$. This fluid model relies on the assumption of a unique value of the radial velocity at each position $r$. However, the nonlinear evolution of the system may lead to multivalued electron velocities with breakdown of the laminar model. Therefore, the fluid treatment will be supplemented with the particle-in-cell model that adequately describes the dynamics of the system in phase space $(r, v)$.

It is convenient to introduce the following nondimensional quantities: $u=v / v_{b}, \quad x=r / R_{e}, \varphi=2 e \phi / m v_{b}^{2}, T$ $=v_{b} t / R_{e}, \nu=n r^{2} / A$, and $\mathcal{E}=-\partial \varphi / \partial x$. The new variable $\nu$ is $x^{2}$ times the density normalized to its value at the emitter. The equations can then be written as

$$
\begin{aligned}
& \left(\frac{\partial}{\partial T}+u \frac{\partial}{\partial x}\right) u=-\frac{1}{2} \mathcal{E}, \\
& \left(\frac{\partial}{\partial T}+u \frac{\partial}{\partial x}\right) \nu=-\nu \frac{\partial u}{\partial x}, \\
& \frac{\partial\left(x^{2} \mathcal{E}\right)}{\partial x}=2 \mu^{2}(1-\nu) .
\end{aligned}
$$

The boundary conditions for the velocity and the density are now expressed as $u(1)= \pm 1$ and $\nu(1)=1$, respectively, and the condition for the electric field, $\mathcal{E}$, is $\int_{x_{i}}^{1} \mathcal{E} d x=0$. While planar Pierce diodes are governed by a single nondimensional number $\alpha$, the behavior of spherical diodes, as reflected in Eqs. (1)-(3) and the boundary conditions, depends on two parameters: $x_{i}=R_{i} / R_{e}$ and $\mu=R_{e} \omega_{p} / v_{b}$, where $\omega_{p}$ is the plasma frequency at the emitter density. This is also true for cylindrical electrodes. ${ }^{17}$

For comparisons with planar devices, typified by the parameter $\alpha$, a number, $\alpha_{c}=\left|x_{i}-1\right| R_{e} \omega_{p} / v_{b}=\left|x_{i}-1\right| \mu$, for diodes with curvature may be used, instead. The steady state with $u_{0}= \pm 1, \nu_{0}=1$, and $\mathcal{E}_{0}=0$, is a basic solution of Eqs. (1)-(3), characterized by constant velocity and no electric field.

\section{B. Electrostatic structures}

In general, steady state solutions (denoted by a subindex 0 ) also include electrostatic structures with nonuniform velocity and nonzero electric field, given by the following nonlinear equation for the potential,

$$
\frac{d}{d x}\left(x^{2} \frac{d \varphi_{0}}{d x}\right)=2 \mu^{2}\left(\frac{1}{\sqrt{1+\varphi_{0}}}-1\right),
$$

the relationships

$$
u_{0} \nu_{0}=1, \quad u_{0}^{2}=\varphi_{0}+1,
$$

and the corresponding boundary conditions. As we shall see in the simulations, when these states are stable, the system tries to evolve towards these configurations.

Let us consider for the moment the small amplitude version of (4), describing states that are close to the $\varphi_{0}=0$ solution,

$$
\frac{d}{d x}\left(x^{2} \frac{d \varphi_{0}}{d x}\right)+\mu^{2} \varphi_{0}=0 .
$$

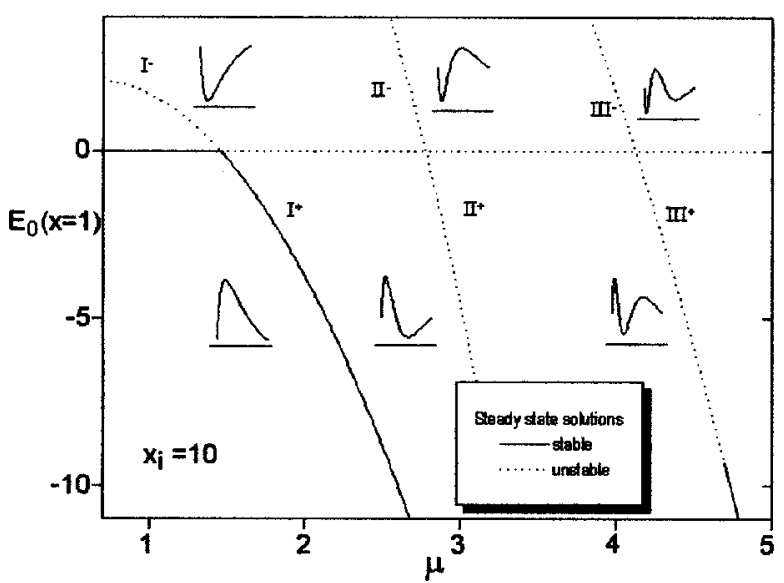

FIG. 2. Bifurcation diagram of the steady states for $x_{i}=10$. The electric field at the emitter, $E_{0}$ vs $\mu$. The shape of the potential in each region and the stability of the branches are also shown.

Equation (5) is an Euler equation with solutions $\varphi_{0}$ that are linear combinations of $x^{\lambda_{1}}$ and $x^{\lambda_{2}}$, where $\lambda_{1,2}=-1 / 2 \pm i d$, with $d=\left(\mu^{2}-1 / 4\right)^{1 / 2}$. Applying the conditions for grounded electrodes we find $x_{i}^{2 i d}=1$, which is equivalent to

$$
\mu_{n}=\left[1 / 4+\left(n \pi / \ln x_{i}\right)^{2}\right]^{1 / 2}, \quad n=1,2,3, \cdots
$$

for $n=1,2,3, \ldots{ }^{16}$ Note that $x_{i}$ and $1 / x_{i}$ give the same $\mu_{n}$ values. Since the square root in (6) must be real, small amplitude solutions exist for $\mu>1 / 2$ only. The sequence of critical values $\mu_{1}, \mu_{2}, \mu_{3}, \ldots$, in whose neighborhood constant and small amplitude modulated velocity steady states coexist, is of great importance for the analysis of the stability and nonlinear dynamics of Pierce diodes.

\section{Bifurcations and stability}

As shown in Ref. 18, in spherical diodes electrostatic structures bifurcate from the simple flows with constant velocity at the critical values $\mu_{n}$ given by (6) in terms of $x_{i}$. Linear perturbations of the steady states, with a time dependence $\exp (s T)$ and amplitudes, $\varphi_{1}, \nu_{1}$, and $u_{1}$, satisfy

$$
\begin{aligned}
& \frac{d}{d x}\left(x^{2} \frac{d \varphi_{1}}{d x}\right)=2 \mu^{2} \nu_{1}, \quad \frac{d}{d x}\left(u_{0} u_{1}-\frac{1}{2} \varphi_{1}\right)=-s u_{1}, \\
& \frac{d}{d x}\left(u_{0} \nu_{1}+\nu_{0} u_{1}\right)=-s \nu_{1} .
\end{aligned}
$$

The steady states and their corresponding perturbations were evaluated using numerical codes based on spectral $\tau$ methods. ${ }^{19}$

A summary of numerical results of the stability analysis for a case of divergent beams, $x_{i}=10$ and $\mu<5$ is presented in Fig. 2. The shape of the potential in each region is shown. The first three branches of nonuniform velocity states intersect the $\mu$ axis at the critical points $\mu_{1}=1.4531, \mu_{2}$ $=2.7742$, and $\mu_{3}=4.1236$. Stable (full lines) and unstable (dotted lines) branches are presented. For $\mu<1 / 2$ (not shown), the constant velocity states are always stable, independent of the value of $x_{i}$. This stability region extends up to $\mu=\mu_{1}$. At $\mu_{1}$ an exchange of stability takes place between modes of uniform and nonuniform velocity states. The 


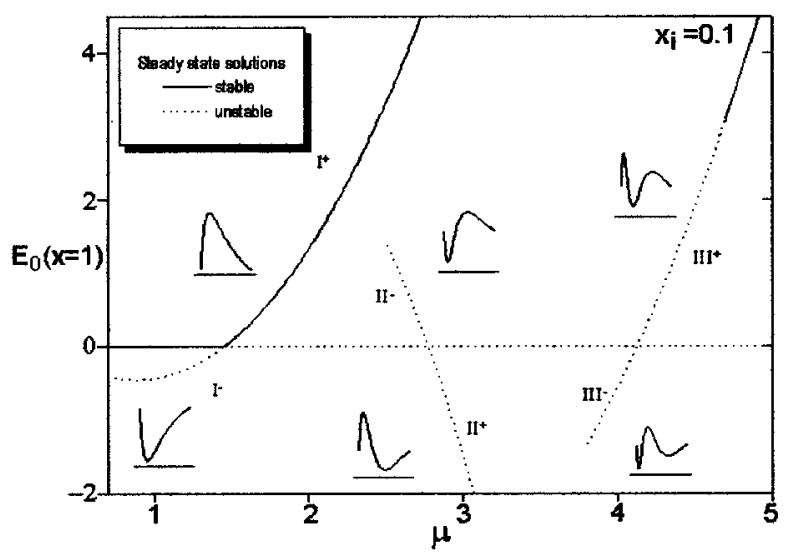

FIG. 3. Same as Fig. 2, for the convergent case, $x_{i}=0.1$.

branch of solutions where the potential has one maximum, identified by $\mathrm{I}^{+}$in Fig. 2, exists for all $\mu>\mu_{1}$ and these configurations are stable. In the interval $2.4<\mu<3.4$ a new branch of solutions (labeled $\mathrm{II}^{-}$and $\mathrm{II}^{+}$) exists. These nonlinear structures with one maximum and one minimum are unstable. The next branch $\left(\mathrm{III}^{-}\right.$and $\mathrm{III}^{+}$), in the interval $3.5<\mu<5.1$, contains structures with two minima and one maximum $\left(\mathrm{III}^{-}\right)$, or two maxima and one minimum $\left(\mathrm{III}^{+}\right)$ of the potential. In this branch, the points $\mu_{H 1}=3.838, \mu_{H 2}$ $=4.694$, and $\mu_{H 3}=4.899$, are Hopf bifurcation points where pulsating electrostatic structures are viable. For higher values of $\mu$ there is a succession of branches in intervals comprising the critical values $\mu_{n}$. All branches, with the exception of $\mathrm{I}^{+}$, exist for a restricted range of $\mu$ values. Their presence is limited by the formation of a virtual cathode at some point inside the diode, where $\varphi \rightarrow 1$. Besides the states with one maximum of the potential that exist for $\mu>\mu_{1}$ and are stable, there is one additional stability region in the interval $\mu_{H 2}<\mu<\mu_{H 3}$ (see the lower right-hand corner of Fig. 2), corresponding to structures where the potential has two maxima.

A similar analysis for the convergent case is presented in Fig. 3, showing a summary of numerical results of stability analysis for $x_{i}=0.1$ and $\mu<5$. The critical values of $\mu$ are the same as for $x_{i}=10$, according to (6).

For a fixed $\mu$ value, the steady state solutions with non uniform velocity for a convergent diode with ratio $x_{i}$ are equivalent to those for a divergent diode with a ratio $x_{i}^{\prime}$ $=1 / x_{i}$. This duality property of solutions, which amounts to an exchange of the roles of emitter and collector (and the sign of the beam velocity) in the pair of diodes considered, can be easily shown considering the invariance of Eq.(4) with respect to a rescaling of the variable $x$ with $x / x_{i}$. Noting, moreover, that for steady states the boundary conditions $u(1)=1$ and $\nu(1)=1$ at the emitter, imply necessarily the conditions $u\left(x_{i}\right)=1$ and $\nu\left(x_{i}\right)=1$ at the collector. This rescaling is an exclusive feature of the spherical geometry, and does not hold for cylindrical diodes. Nevertheless, it must be noted that the value of the electrical field at the emitter is different for $x_{i}=10$ and $x_{i}=0.1$, as a comparison of Figs. 2 and 3 shows.

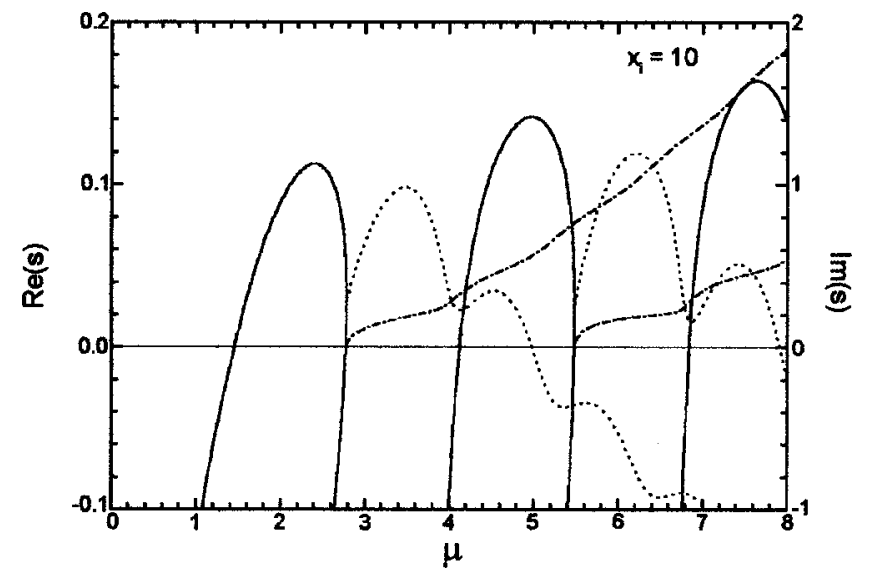

FIG. 4. Roots of the dispersion relation for $x_{i}=10$ as a function of $\mu$ : real roots, full lines; complex roots, $\operatorname{Re}(s)$ : dotted lines and $\operatorname{Im}(s)$ : dasheddotted lines.

It is also interesting to note that calculations show that the eigenvalues of the linear perturbations computed for $x_{i}$ $=10$, both for constant velocity states and for nonuniform velocity states, are also eigenvalues of the $x_{i}=0.1$ case, provided a dimensionless time variable $T /\left|x_{i}-1\right|$, measured in units of the transit time $\tau=R_{e}\left|x_{i}-1\right| / v_{b}$, is employed. In other words, the computed values of $s\left|x_{i}-1\right|$ are the same in the two dual problems. Again, this is a property not observed for cylindrical diodes. ${ }^{17}$

\section{Stability of constant velocity states}

The initial states of the diodes are represented by solutions with $u_{0}= \pm 1$. A linearization of the set of Eqs. (1)-(3) for perturbations of the constant velocity solutions with a time dependence of the form $\exp (s T)$ leads to the dispersion relation

$$
(1 / 2+i d+s) I_{2}-(1 / 2-i d+s) I_{1}+2 i s d e^{s} I_{0}=0,
$$

with the following expressions for $I_{0}, I_{1}$, and $I_{2}$ :

$$
\begin{aligned}
I_{0}= & \frac{1}{2 i d} \int_{x_{i}}^{1} e^{s x}\left(x^{(-3 / 2+i d)} \int_{1}^{x} e^{-s z} z^{(1 / 2-i d)} d z\right. \\
& \left.-x^{(-3 / 2+i d)} \int_{1}^{x} e^{-s z} z^{(1 / 2+i d)} d z\right) d x, \\
I_{1}= & \int_{x_{i}}^{1} e^{s x} x^{(-3 / 2+i d)} d x, \quad I_{2}=\int_{x_{i}}^{1} e^{s x} x^{(-3 / 2-i d)} d x .
\end{aligned}
$$

This equation was derived in Ref. 16, but the roots were examined only near the critical values $\mu_{n}$ given by (6). Here we solved numerically the dispersion relation (7) for the characteristic values $s$, without restrictions. The roots appear as an infinite sequence of branches. We can see a typical display in Fig. 4, which shows real roots (full lines) and complex ones $[\operatorname{Re}(s)$ : dotted lines and $\operatorname{Im}(s)$ : dashed-dotted lines] for $x_{i}=10$, a diverging flow case, as functions of $\mu$, in the range $0<\mu<8$. For negative values of $\operatorname{Re}(s)$ there appear five branches which represent damped modes $[$ with $\operatorname{Im}(s)$ $=0]$. Each of these branches attains the $s=0$ value at $\mu_{1}$ to $\mu_{5}$, respectively, given by $(6)$. The instability, $\operatorname{Re}(s)>0$, first 
appears for $\mu>\mu_{1}$. For a value of $\mu$ slightly larger than $\mu_{2}$ the first and second (real) branches merge and become a pair of complex conjugate branches which are oscillatory unstable up to $\mu \sim 5$ and oscillatory damped thereafter. The third branch becomes unstable for $\mu>\mu_{3}$, and meets a fourth branch for a higher value of $\mu$, repeating the same stability pattern of the first and second branches. The numerical solutions of (7) also show that, for all $\mu$, the quantity $s\left|x_{i}-1\right|$ has the same values for $x_{i}$ and $1 / x_{i}$ diodes. Thus, Fig. 4 can be applied also to the convergent case $x_{i}=0.1$, after rescaling the corresponding $s$ roots.

\section{E. Energy analysis of the stability of flows with uniform velocity}

The Pierce diode is an open system and its energy content varies during unsteady regimes. The instability of constant velocity states can be analyzed in terms of the energy flow balance of the system. This approach sets out the role of the Maxwell radial electric stress power exerted on the electron flow, an effect absent in planar diodes, which acts in opposite ways in divergent or convergent flows. Using (1)(3) and the boundary conditions, it is easy to verify that

$$
\frac{\partial}{\partial T} \int_{1}^{x_{i} x^{2}} \frac{\mathcal{E}^{2}}{2} d x=2 \mu^{2} \int_{1}^{x_{i}} u \nu \mathcal{E} d x
$$

This equation states that the rate of time variation of the electrostatic energy of the diode is due to the power delivered by the electric current to the field.

Computing the product $u \nu \mathcal{E}$ from (1)-(3) as

$$
-2 \mu^{2} \mathcal{E} u \nu=\left(2 \mu^{2}-\frac{\partial}{\partial x}\left(x^{2} \mathcal{E}\right)\right)\left(\frac{\partial}{\partial T}\left(\frac{u^{2}}{2}\right)+u \frac{\partial}{\partial x}\left(\frac{u^{2}}{2}\right)\right)
$$

after some simple manipulations a formula for the change of the total (electrostatic plus kinetic) energy, $E_{T}$,

$$
E_{T} \equiv \int_{1}^{x_{i}}\left(\frac{x^{2}}{2} \mathcal{E}^{2}+2 \mu^{2} \nu \frac{u^{2}}{2}\right) d x
$$

can be derived,

$$
\frac{d E_{T}}{d T}=-2 \mu^{2}\left[\nu u \frac{u^{2}}{2}\right]_{1}^{x_{i}} .
$$

The left-hand-side term of (9) represents the difference between the inflow and outflow of kinetic energy through the boundaries of the system. In nondimensional units the inflow of kinetic energy through the emitter is 1 . For divergent diodes $\nu u>0$ at $x_{i}>1$, while for convergent diodes $\nu u<0$ at $x_{i}<1$, so that in both cases we can write

$$
\frac{d}{d T}\left|E_{T}\right|=\mu^{2}\left[1-|\nu u| \frac{u^{2}}{2}\right]_{x_{i}} \text {. }
$$

Thus, the total energy grows in time when the inflow of kinetic energy is larger than the outflow, and vice versa.

The instability develops when conditions for growth can be steadily maintained in time. We show now how these can be written in the case of the linear perturbations of constant velocity states $u_{0}= \pm 1$. We set $\mathcal{E}_{1}=E_{1}(x) \exp (s T)+$ c.c., with $s=p+i \omega$, where $p, \omega$ are the real and imaginary parts of $s$, respectively, c.c. denotes the complex conjugate of the preceding term, with similar expressions for $u$ and $\nu$. The linearized version of (1) and (3), with subindex 1 denoting perturbation quantities, is

$$
\left(\frac{\partial}{\partial T}+u_{0} \frac{\partial}{\partial x}\right) u_{1}=\frac{1}{2} \mathcal{E}_{1}, \quad \frac{\partial}{\partial x}\left(x^{2} \mathcal{E}_{1}\right)=-2 \mu^{2} \nu_{1} .
$$

Using them in (8), we obtain

$$
\begin{aligned}
& \frac{\partial}{\partial T} \int_{1}^{x_{i}} x^{2}\left(\frac{\mathcal{E}_{1}^{2}}{2}+\frac{2 \mu^{2}}{x^{2}} u_{1}^{2}\right) d x \\
& \quad=-u_{0}\left[x^{2}\left(\frac{\mathcal{E}_{1}^{2}}{2}+\frac{2 \mu^{2}}{x^{2}} u_{1}^{2}\right)\right]_{1}^{x_{i}}-u_{0} \int_{1}^{x_{i}} \frac{\mathcal{E}_{1}^{2}}{2} \frac{\partial}{\partial x}\left(x^{2}\right) d x .
\end{aligned}
$$

We note now that, when $\omega=0$, we may write $\mathcal{E}_{1}^{2}$ $=\left(2 \operatorname{Re}\left(E_{1}\right)\right)^{2} \exp (2 p T)$ and $(\partial / \partial T) \mathcal{E}_{1}^{2}=2 p \mathcal{E}_{1}^{2}$, with similar expressions for $u_{1}^{2}$. If, instead, we have $\omega \neq 0$ but the growth is small over an oscillation period, $\omega>>|p|$, then by time averaging over one period we get $\left\langle\mathcal{E}_{1}^{2}\right\rangle=2\left|E_{1}\right|^{2} \exp (2 p T)$. Thus, for this case the same results of the case with $\omega=0$ are obtained, replacing $\left(\operatorname{Re}\left(E_{1}\right)\right)^{2}$ by $\left|E_{1}\right|^{2}$. Therefore, in both cases we find

$$
p=\frac{u_{0}\left[x^{2}\left(\left|E_{1}\right|^{2}+\frac{4 \mu^{2}}{x^{2}}\left|u_{1}\right|^{2}\right)\right]_{x_{i}}^{1}-\int_{1}^{x_{i}}\left|E_{1}\right|^{2} \frac{\partial}{\partial x}\left(x^{2} u_{0}\right) d x}{2 \int_{1}^{x_{i}} x^{2}\left(\left|E_{1}\right|^{2}+\frac{4 \mu^{2}}{x^{2}}\left|u_{1}\right|^{2}\right) d x} .
$$

This formula gives the growth (or damping) rate as the ratio of the difference of the net energy inflow and the power of the radial electric stress component over 4 times the total energy content of the perturbative mode. While the denominator is positive definite, the numerator can be positive or negative. The last term in the numerator of (10) represents the power due to the radial electric tension during the expansion or compression of spherical shell volumes, in divergent or convergent flows, respectively. In divergent diodes, $x_{i}$ $>1, u_{0}=+1$, this term is negative and it always provides a stabilizing contribution. In convergent diodes, $x_{i}<1$, $u_{0}=-1$, the power of the electric stress component gives always a destabilizing contribution, instead. In short, when a material volume convected by the flow increases, as it happens in divergent diodes, perturbative electrostatic energy is spent during the expansion. The opposite happens in convergent diodes. The numerical experiments reported in Sec. V give evidence of differences in the evolution of convergent and divergent diodes after a laminar flow breaks down, even when dual $x_{i}$ and $1 / x_{i}$ diodes are compared.

\section{PARTICLE-IN-CELL MODEL}

Particle simulation techniques are by now a well established and effective technique for studying the nonlinear properties of plasmas. Unlike the fluid model that is based on the assumption of a unique value of the radial velocity at every position, particle simulations allow multivalues of velocities originated by the overcrossing of different elements. 
The basic concepts involved in particle simulations are well known. ${ }^{20}$ In principle, we follow the movement of a large number of particles under the action of collective (selfconsistent) and externally applied electromagnetic fields. At time $t=0$ we know the velocities and positions for each particle and from these we obtain charge and current densities. These are then used to derive the electromagnetic field. Using these fields we advance the positions and velocities, and a new cycle starts.

The particle code used here is PDS1 (plasma device spherical one dimensional). ${ }^{21}$ This code is one-dimensional, radial, electrostatic, and simulates a plasma contained between concentric spheres. The spherical grids can be coupled to an external RLC (resistive, inductive, and/or capacitive) circuit and/or rf source, i.e., it is not specialized to the problem treated here. During the simulations the applied boundary conditions ensure that electrons are injected at a constant rate from the emitter (inner electrode for a divergent beam, $x_{i}>1$, and outer electrode for a convergent beam, $x_{i}<1$ ). When electrons reach the collector they are absorbed, and contribute to the external surface charge. ${ }^{21}$ Both electrodes are kept at the same potential, $\phi=0$. Fields are calculated on a grid of 400 points using a finite-difference Poisson scheme (equivalent to the flux conserving method ${ }^{20}$ ). Poisson's equation is solved in each time step with the given boundary conditions. More details about PDS1 can be obtained in Refs. 21-23.

The time step is determined by ensuring that the Courant-Friedrichs-Levy condition $v_{\max } \Delta t / \Delta x<1$ is satisfied. A value of $v_{\max }=3 v_{b}$ is assumed, after concluding from a series of numerical experiments that this is an upper bound for the velocity. This condition means that no disturbance can propagate more than $\Delta x$ in a time $\Delta t$, and avoids numerical instabilities.

The original code has been modified in order to have an initial density that varies as $n_{0} \propto 1 / r^{2}$. The algorithm for this distribution was obtained as follows (see Ref. 20, p. 388). We wish to place particles in phase space so as to form a density $n(r)=A / r^{2}$, from $r=r_{a}$ to $r=r_{b}, A$ being a constant. We form a cumulative distribution function

$$
N(r) \equiv \frac{\int_{r_{a}}^{r} n\left(r^{\prime}\right) d r^{\prime}}{\int_{r_{a}}^{r_{b}} n\left(r^{\prime}\right) d r^{\prime}},
$$

where $N\left(r_{a}\right)=0, N\left(r_{b}\right)=1$, and

$$
\frac{d N(r)}{d r}=\frac{n(r)}{\int_{r_{a}}^{r_{b}} n\left(r^{\prime}\right) d r^{\prime}} .
$$

We see that equating $N\left(r_{s}\right)$ to a uniform distribution of numbers $R_{s}, 0<R_{s}<1$ will produce the $r_{s}$ corresponding to the distribution $n\left(r_{s}\right)$.

The total number of particles is given by

$$
N_{\text {total }}=\int_{0}^{2 \pi} \int_{0}^{\pi} \int_{r_{a}}^{r_{b} A} \frac{r^{2}}{r^{2}} \sin \theta d \theta d \varphi d r=4 \pi A\left(r_{b}-r_{a}\right) .
$$

Between one particle and the following $N_{\text {total }}=1$, and we can write

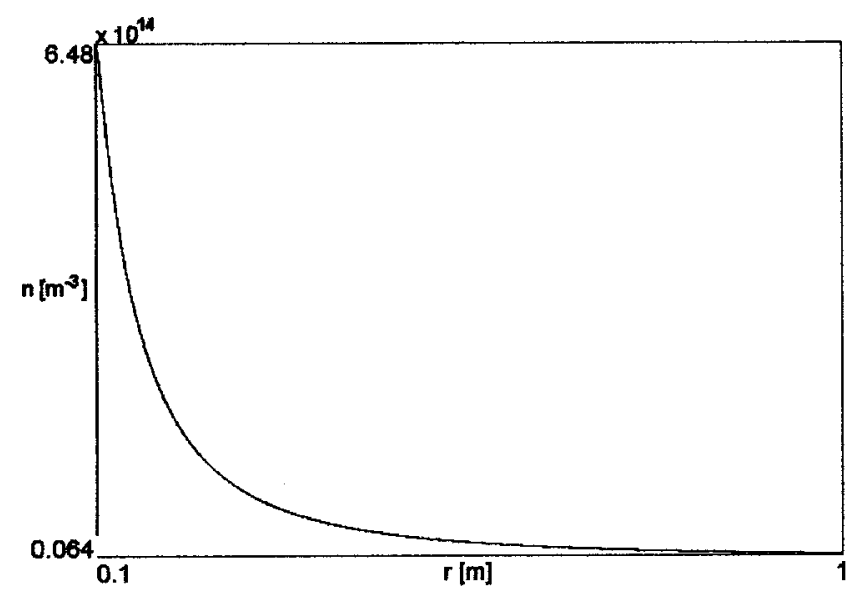

FIG. 5. Example of initial density profile.

$$
\frac{1}{N_{\text {total }}}=\frac{4 \pi A\left(r_{i+1}-r_{i}\right)}{4 \pi A\left(r_{b}-r_{a}\right)}=\frac{\left(r_{i+1}-r_{i}\right)}{r_{b}-r_{a}},
$$

$r_{i}$ being the position of $i$ th particle. We can now find the position of the first particle

$$
r_{1}=r_{a}+\left(r_{b}-r_{a}\right) /\left(N_{\text {total }}\right) .
$$

Observe that the last term in (11) is a constant that we call $N_{p}$. We can now write that $r_{i+1}=r_{i}+N_{p}$, and we have the positions of all particles in the simulations, for $t=0$. It is important to keep in mind that $N_{\text {total }}$ is the total number of macroparticles to be used in the simulation. Results of this algorithm are shown in Fig. 5, for $x_{i}=10$. For reference, all runs discussed in this paper were standardized to involve some 70000 simulation particles (macroparticles), the precise number varying during each run.

Initial conditions play a very important role in nonlinear problems. In the case treated here, the idea is to start the simulation with $n_{e}(r, t=0)=n_{i}(r, t=0)$, which gives $\phi(r, t$ $=0) \simeq 0$, and the electric field at the emitter, $E_{0}$, also zero except for a very small numerical noise. In general, results for the stability of the constant velocity states obtained with this initialization agree well with those obtained with fluid models. However, in some instances, it is necessary to initialize the system with a nonzero potential to reach the desired stable state. In that case the initial perturbation affects the value of $A$ only, and does not entail a change of the density radial dependence, that remains $1 / r^{2}$, or in the flow velocity. It is important to emphasize that the perturbation is imposed at $t=0$, and leaves the system after one transit time.

\section{NUMERICAL SIMULATION RESULTS}

In this section we present particle simulation results obtained for $x_{i}=10$, divergent case, and its dual convergent case, $x_{i}=0.1$, for different values of $\mu$, testing the theoretical predictions and stability diagrams of Sec. III. Investigations for other values of $x_{i}$, starting close to 1 where the properties of the spherical diodes converge to those of planar devices, up to values of $x_{i}$ much larger and much smaller than 1 where curvature effects are relevant (like the ones discussed in this section), have shown nothing qualitatively dif- 

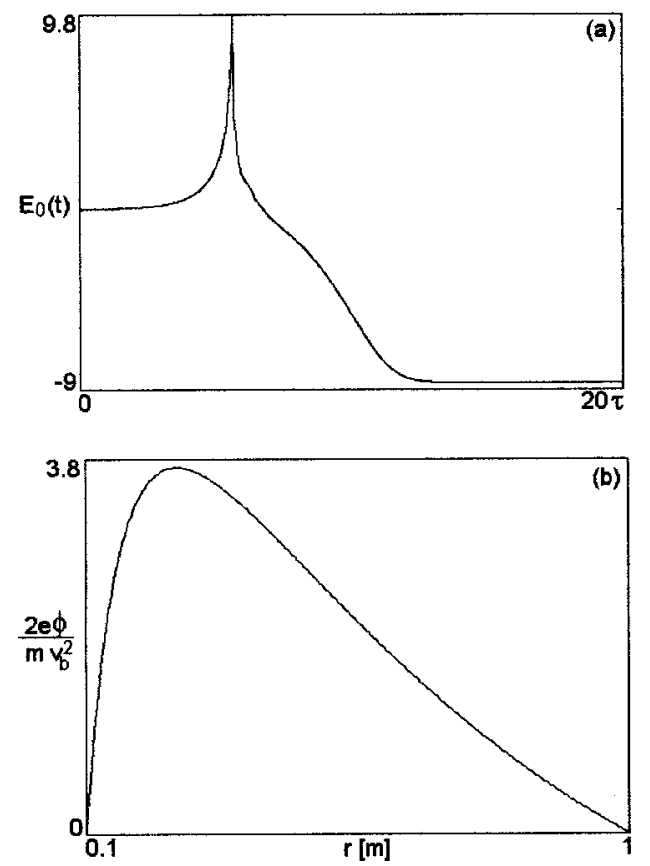

FIG. 6. Results for $x_{i}=10$, and $\mu=2.5$. (a) Time evolution of the electric field at the emitter, up to $t=20 \tau$; (b) space profile of potential at $t=20 \tau$.

ferent in matters of bifurcations or dynamics. All numerical experiments start from the basic constant velocity states, and are initialized as described in Sec. IV.

\section{A. The divergent case}

For $x_{i}=10$ we started by simulating the system with $\mu$ $<1 / 2$. We chose $\mu=0.4$, and several initial perturbations of space charge with $\phi \neq 0$. In all cases the simulations have shown that the system is stable, the perturbations decay, and a final state with $v=v_{0}$ is reached. This is in agreement with the linear stability analysis.

We have verified that the constant velocity states are unstable for $\mu>1.45=\mu_{1}$. For $\mu$ larger than the first critical value simulations show that the potential approaches the electrostatic solution with one maximum corresponding to the branch $\mathrm{I}^{+}$of Fig. 2 . The same figure indicates that for values of $\mu$ in the interval $2.4<\mu<3.4$, branch II, it should be possible to observe two different behaviors, depending on the value of $E_{0}$. We have done simulations for several values of $\mu$ in this interval. As an example, we show results for $\mu=2.5$. The system departs from the uniform velocity configuration and approaches a new stable state with nonuniform velocity, independent of the initialization. Figure 6(a) shows the time evolution of $E_{0}$ up to $20 \tau$. It seems that the system starts evolving towards the unstable state of branch $\mathrm{II}^{-}$of Fig. 2 by increasing $E_{0}$ to large positive values. In fact, the peak in $E_{0}$ observed at a time $\simeq 6 \tau$, is a manifestation of a virtual cathode formation. As the system evolves in time it relaxes to a stable state with $E_{0} \simeq-8.8$, and a potential with one maximum as shown in Fig. 6(b) which corresponds to branch $\mathrm{I}^{+}$. Notice that the signs of $E_{0}$ are opposite for branch $\mathrm{II}^{-}$(unstable) and branch $\mathrm{I}^{+}$(stable) solutions, in the interval $2.4<\mu<\mu_{2}=2.7742$. Summarizing, for this set of parameters the numerical experiment
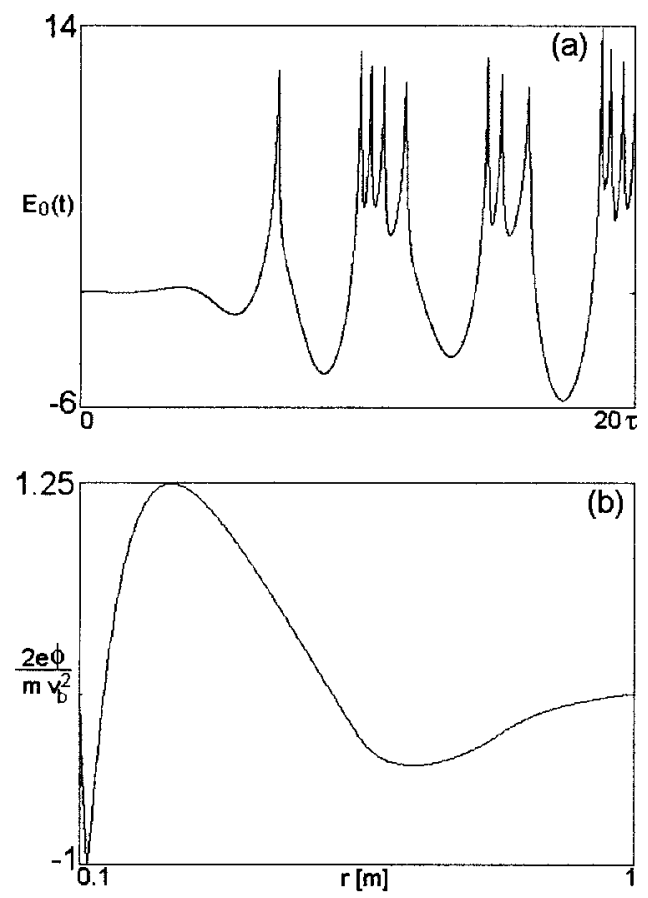

FIG. 7. Results for $x_{i}=10$, and $\mu=3.4$. (a) Time evolution of $E_{0}$ up to $t$ $=20 \tau$; (b) space profile of potential at $t=20 \tau$.

shows that, (a) an unstable electrostatic structure is approached, (b) the laminar flow is disrupted by the formation of a virtual cathode, (c) a transient stage follows in which the system spontaneously eliminates an excess of electrons and recomposes a laminar flow, and (d) finally the diode settles down to a stable nonuniform velocity state.

As we increase the value of $\mu$, we find similar results. New features appear for the case $\mu=3.4$, the upper limit of the interval. Figure 7 (a) shows the time evolution of $E_{0}$ up to $20 \tau$. The peaks are related to the virtual cathode formation, while the minima are related to the presence of a laminar flow. Figure 7(b) shows the space profile of the potential at $t=20 \tau$, an unstable solution. Here, the diode shows an intermittent sequence of disruptions and recoveries of the laminar flow, which does not seem to stop.

We have also simulated values of $\mu$ close to the Hopf bifurcation points. We present now results for three different values of $\mu: 4.5, \mu_{H 2}$, and 4.8. Figure 8 describes the dynamics of the system for $\mu=4.5$. For this value of $\mu$ there are no stable laminar flows and the dynamics becomes more complex. Figure 8(a) shows the time evolution up to $25 \tau$ of the electric field at the emitter $\left(E_{0}\right)$. Near $\tau=7$ a state of type $\mathrm{III}^{+}$is formed as shown in Fig. 8(b). The system oscillates near this unstable state, albeit with increasing amplitude, as shown for $E_{0}$ from $t=25 \tau$ to $46 \tau$ in Fig. 8(c). The departure of the system from this unstable configuration is illustrated in Fig. 8(d) where a phase space portrait, $d E_{0} / d t$ vs $E_{0}$, is given for the same time interval of Fig. 8(c). The time evolution of $E_{0}$ from $t=50 \tau$ to $75 \tau$, after the break up of the laminar behavior is presented in Fig. 8(e). At that point the electric field oscillations contain a mixture of frequencies in contrast with the previous situation in the time interval 7-30 $\tau$ where a well-defined frequency was observed. The 

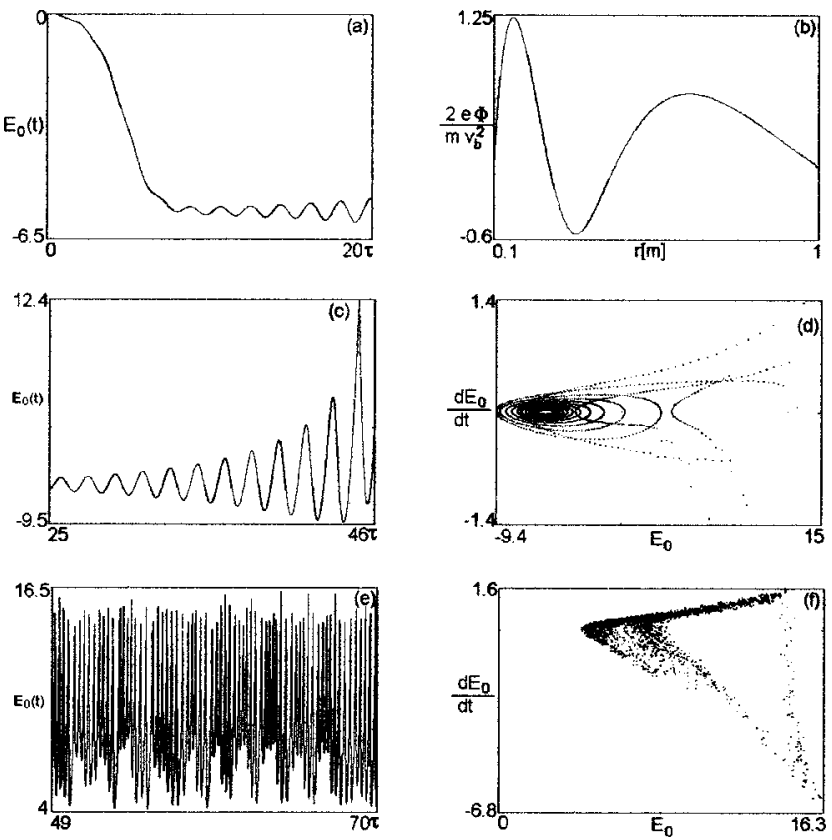

FIG. 8. Results for $x_{i}=10$ and $\mu=4.5$. (a) Time evolution of $E_{0}$ up to $t$ $=20 \tau$; (b) potential profile at $t=20 \tau$; (c) time evolution for $E_{0}$ for a later time, $25 \tau<t<46 \tau$; (d) the corresponding phase space $d E_{0} / d t$ vs $E_{0}$; (e) time evolution for $E_{0}$ for $49 \tau<t<70 \tau$; and (f) the corresponding phase space $d E_{0} / d t$ vs $E_{0}$.

phase space of Fig. 8(f) corresponding to the same time lapse of Fig. 8(e) depicts the complexity of this stage of the evolution. The phase space of electrons, $v$ vs $r$ (not shown), for this last period indicates intermittent formation of virtual cathode and generally shows overcrossing of electron motions.

When $\mu=4.8$, a stable $\mathrm{III}^{+}$state is possible in principle. However, the evolution found by particle simulations depends on the initial conditions. Stable solutions are found if we start with $n_{e}<n_{i}$, which gives the potential shown in Fig. 9(a). After $t=25 \tau$ the observed potential is shown in Fig. 9(b). The shape of the potential is clearly identified with the steady state solution $\mathrm{III}^{+}$and the average value of $E_{0}$ is in
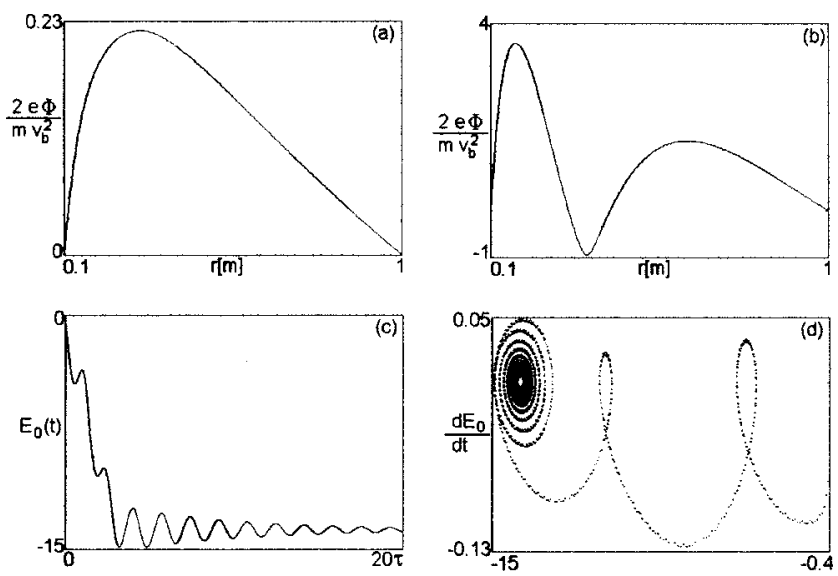

FIG. 9. Results for $x_{i}=10$ and $\mu=4.8$. (a) Initial potential profile; (b) potential at $t=20 \tau$; (c) time evolution of $E_{0}$, showing damped oscillations; (d) phase space, $d E_{0} / d t$ vs $E_{0}$, showing a circle converging to a point.
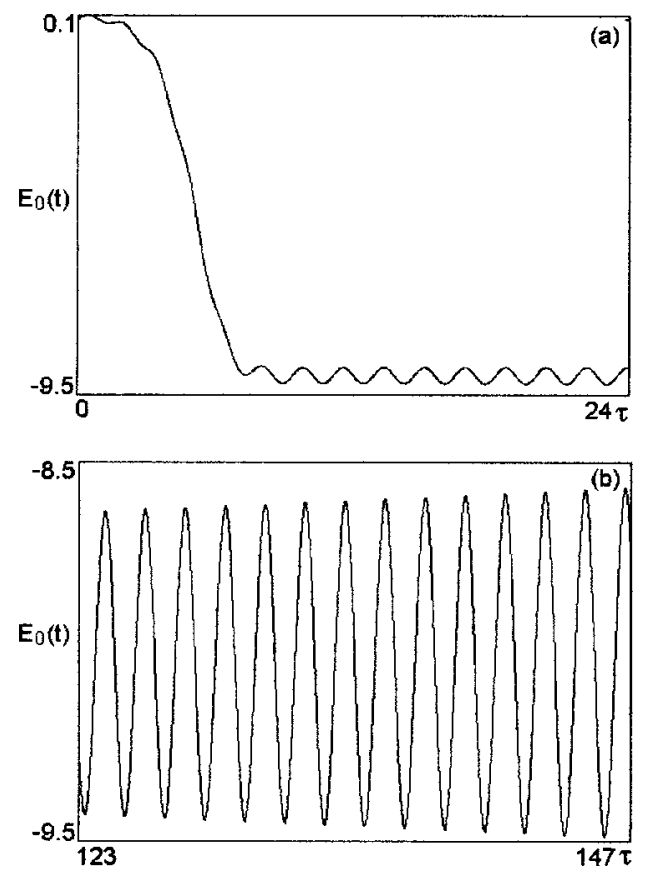

FIG. 10. Results for $x_{i}=10$ and $\mu=\mu_{H 2}=4.694$. (a) Time evolution of $E_{0}$ up to $t=24 \tau$; (b) time evolution of $E_{0}$ after stabilization, $123 \tau<t<147 \tau$.

good agreement with the value of Fig. 2. The time evolution of $E_{0}$ is presented in Fig. 9(c) and the corresponding phase space, $d E_{0} / d t$ vs $E_{0}$, showing cycles converging to a center, is presented in Fig. 9(d). The damping of $E_{0}$ towards the asymptotic steady state reaching a final value of -13.8 is clear.

According to theory we expect nonlinearly oscillatory states for $\mu$ close to $\mu_{H 2}$. In fact, the simulations for $\mu$ $=4.694$ confirm this behavior. Figure 10(a) gives the time evolution of $E_{0}$ up to $t=24 \tau$, where we can see that the value of $E_{0}$ goes from a small perturbation to oscillations around a value close to -9.0 . After a long simulation time an interval 123-147 $\tau$ is shown in Fig. 10(c) where one can see that the electric field is still oscillating around the average -9.0 with a well-defined frequency. The phase space $d E_{0} / d t$ vs $E_{0}$ (not shown here) portraits a limit cycle. The electrons maintain a laminar flow throughout the steady state oscillations.

\section{B. The convergent case}

We consider now the convergent case, $x_{i}<1$. We have selected $x_{i}=0.1$, the dual of the previously discussed divergent case $x_{i}=10$. The linear stability analysis is summarized in Fig. 3. We have done particle simulations for different values of $\mu$, keeping $x_{i}=0.1$. For values of $\mu<\mu_{1}$, several numerical experiments have been carried out and the initial constant velocity states were found to be stable, confirming the predictions of the linear theory.

For values of $\mu$ in the range $2.4<\mu<3.4$, the simulations show that the evolution of the diode depends on the initial conditions. This is illustrated with an example for $\mu$ $=2.5$. When we start the simulation with $n_{e}(r, t=0)$ slightly larger than $n_{i}(r, t=0)$, the system becomes unstable and the 

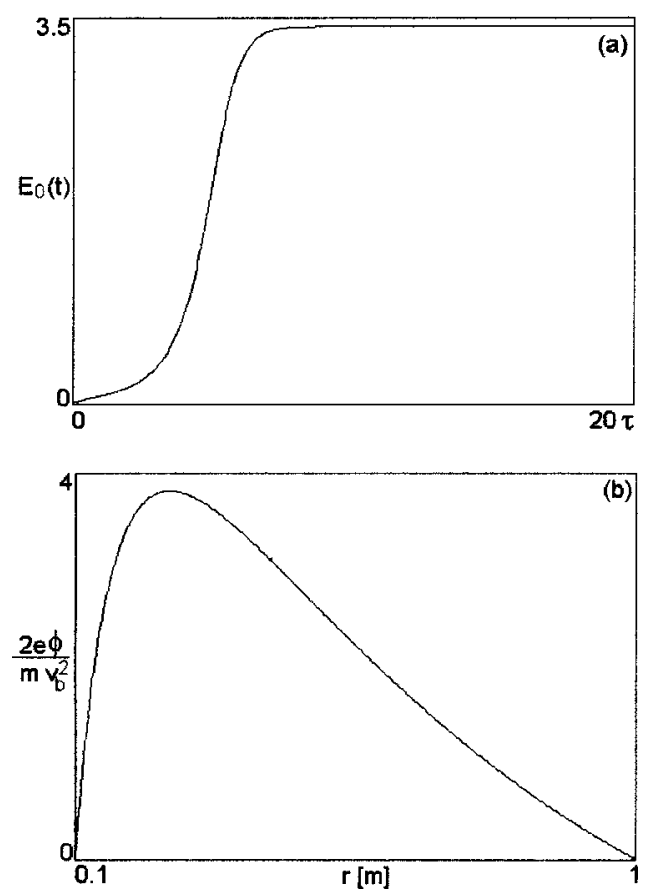

FIG. 11. Results for $x_{i}=0.1$, and $\mu=2.5$ with initial condition $\phi=0.0$. (a) Time evolution of the electric field at the emitter; $E_{0}$ goes to a stable value of $E_{0}=\sim 3.41$. (b) Space profile of the potential at $t \simeq 20 \tau$.

laminar flow is disrupted. A virtual cathode is formed near the emitter and remains during the period examined. If, instead, we start the simulation with $n_{e} \simeq n_{i}$ so that the initial potential is close to zero, the evolution of the system approaches a stable nonuniform velocity state. This is illustrated in Fig. 11(a) that shows the time evolution of the electric field at the emitter (the outer electrode for convergent beams). The electric field at the emitter approaches asymptotically the value $E_{0} \simeq 3.41$. The potential presents one maximum as in solutions of type $\mathrm{I}^{+}$in Fig. 3. The potential is shown in Fig. 11(b) for $t \simeq 20 \tau$. Other values of $\mu$ in the same interval have been examined with similar results.

We have also run simulations for $\mu=3.8$ where we found that the system is unstable and the laminar flow breaks down. Some features of the electron phase space will be discussed in the last section.

The next selected value of $\mu$ was $\mu_{H_{2}}$, a Hopf bifurcation point. Particle simulations reveal the presence of an oscillatory stable state, as we can see in Fig. 12. Figures 12(a)12(b) show the time evolution of the electric field at the emitter for the intervals $0<t<20.5 \tau$, and $20.5 \tau<t<40 \tau$, respectively. The electric field $E_{0}$ grows in time until steady state oscillations are attained. Figure 12(c) shows the space profile of the potential at $t=40 \tau$, presenting the same shape indicated in Fig. 3 for branch $\mathrm{III}^{+}$. Figure 12(d) shows the spectrum of the electric field at the emitter, with a welldefined peak at the frequency, $f=1.9 \times 10^{7} \mathrm{~Hz}$, which in nondimensional units is $\operatorname{Im}\{\lambda\}=0.4$, in good agreement with fluid theory. We have changed the value of $\mu$ to $\mu=4.7$ and results are similar.

We have performed particle simulations for $\mu=4.8$, and found that a nonuniform velocity state is approached asymptotically. Figure 13 shows the results. Figure 13(a) presents
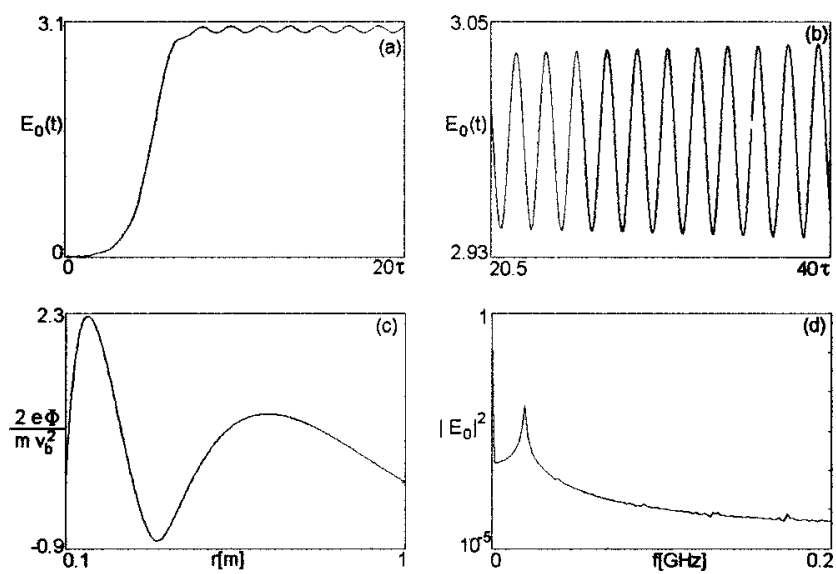

FIG. 12. Results for $x_{i}=0.1$, and $\mu=\mu_{H 2}$. Time evolution of the electric field at the emitter for the intervals (a) $0<t<20 \tau$ and (b) $20 \tau<t<40 \tau$. (c) Space profile of the potential at $t=40 \tau$. (d) Spectrum of the electric field at the emitter, with one characteristic frequency, $f=1.9 \times 10^{7} \mathrm{~Hz}$, which in nondimensional units is 0.4 .

the time evolution of the electric field at the emitter up to $t$ $=20 \tau$. The damping of $E_{0}$ to the constant value of $E_{0}$ $\sim 4.5$, is apparent. Figure 13(b) shows the potential at $t$ $=20 \tau$, with two maxima as predicted by fluid model. In fact, Fig. 3 indicates the presence of stable solutions for the region $\mu_{H 2}<\mu<\mu_{H 3}$.

The simulations confirm the theoretical idea embodied in the duality property of the spherical diodes showing that the potential profile of Fig. 13(c) $\left(x_{i}=0.1\right)$ is equal to the potential profile of Fig. 9(b) $\left(x_{i}=10\right)$ for the same value of $\mu$. However, a different behavior can be observed in the 2.4 $<\mu<3.4$ interval where the initial state is unstable and two electrostatic states (one, $\mathrm{I}^{+}$, stable, the other, $\mathrm{II}^{-}$, unstable)
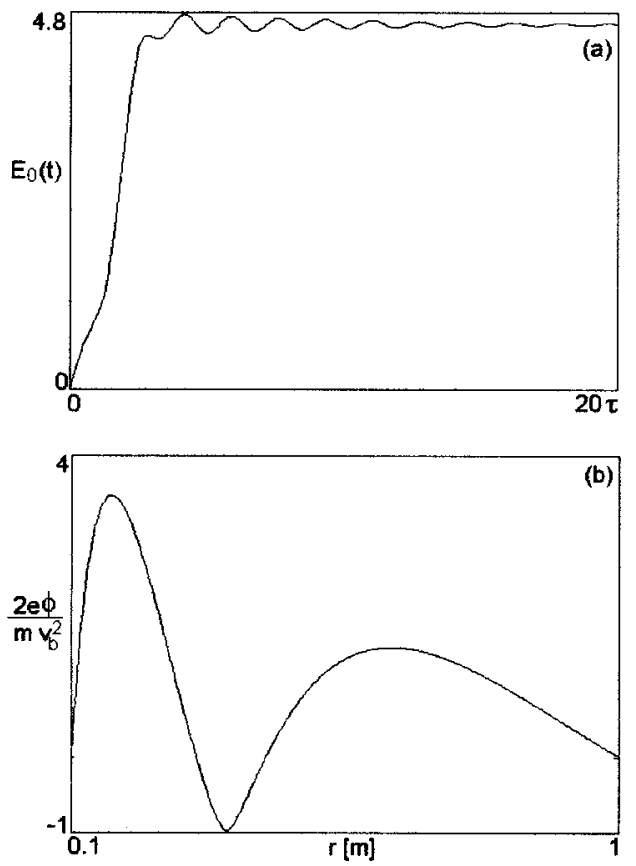

FIG. 13. Results for a particle simulation with $x_{i}=0.1$, and $\mu=4.8$. (a) Time evolution of the electric field at the emitter up to $t=20 \tau$. (b) Potential at $t=20 \tau$. 

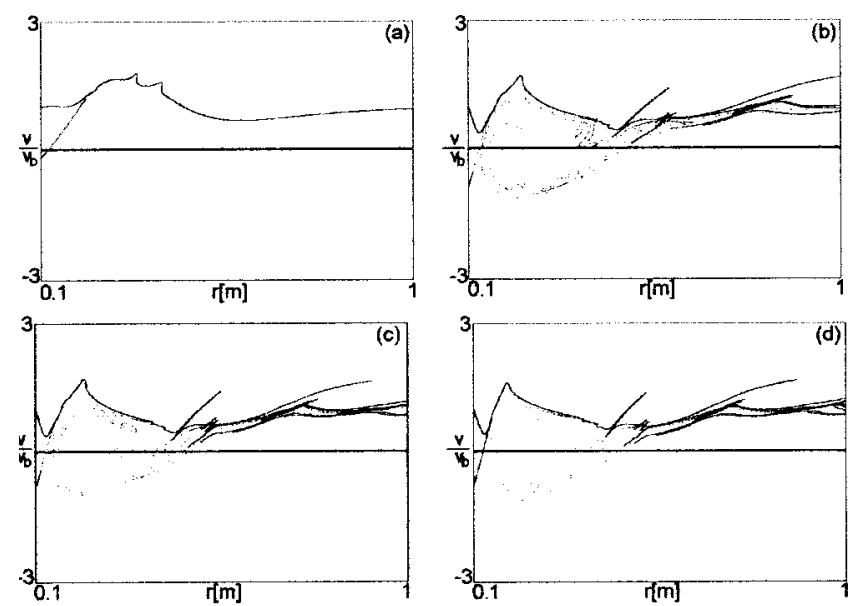

FIG. 14. Phase space, $v$ vs $r$, at different times, for $x_{i}=10$ and $\mu=3.8$. (a) $t=5 \tau$, (b) $t=10 \tau$, (c) $t=20 \tau$, and (d) $t=60 \tau$.

exist: convergent diodes may evolve either to a new laminar state or toward turbulence, depending on the initialization, while divergent diodes show loss of laminarity followed by spontaneous recovery of a laminar state no matter how the simulation is initialized.

\section{Phase space dynamics}

We examine now the disruption of the laminar flow in convergent and divergent systems, using as a diagnostic method the phase space $v$ vs $r$. We have chosen two dual systems, $x_{i}=10$ and 0.1 , for comparison. Both simulations are performed at $\mu=3.8$. In Fig. 14 the four panels (a)-(d) are phase space portraits at $5,10,20$, and $60 \tau$, respectively, for $x_{i}=10$. The system was started at a constant velocity state, taking the numerical noise as initial perturbation. The line at $v / v_{b}=0$ represents the neutralizing background ions with a fixed $1 / r^{2}$ density profile. In Fig. 14(a), at $5 \tau$ we see that the laminar flow has already been disrupted and that a weak virtual cathode has been formed near the emitter, reflecting a small fraction of the incoming electrons. An acceleration process, hinted by the two velocity spikes near the emitter, is in its initial stage. Five transit times later this process is fully developed and a few jets of energetic particles are now present.

The formation of jets starts near the virtual cathode so that, for instance, the peak seen in Fig. 14(b) will develop into a jet traveling away from the emitter. The jets are accompanied by streams of electrons whose velocity is being slowed down, as can be clearly seen in Fig. 14(b). Some of these electrons can be reflected and then form part of a small population of trapped particles near the emitter. The structures seen at $10 \tau$ will be convected out of the system, but new jets are continuously being formed, reproducing the same general pattern, as seen at $20 \tau$.

The configuration at $20 \tau$ is similar to the one at $10 \tau$, but the two stages are unrelated in the sense that the jets seen in these two plots are formed by groups of electrons entering the system at different times. The repetition of these patterns is quasi-periodic as can be seen by comparing the phase space at $60 \tau$ with that at $20 \tau$, and observing that the fine
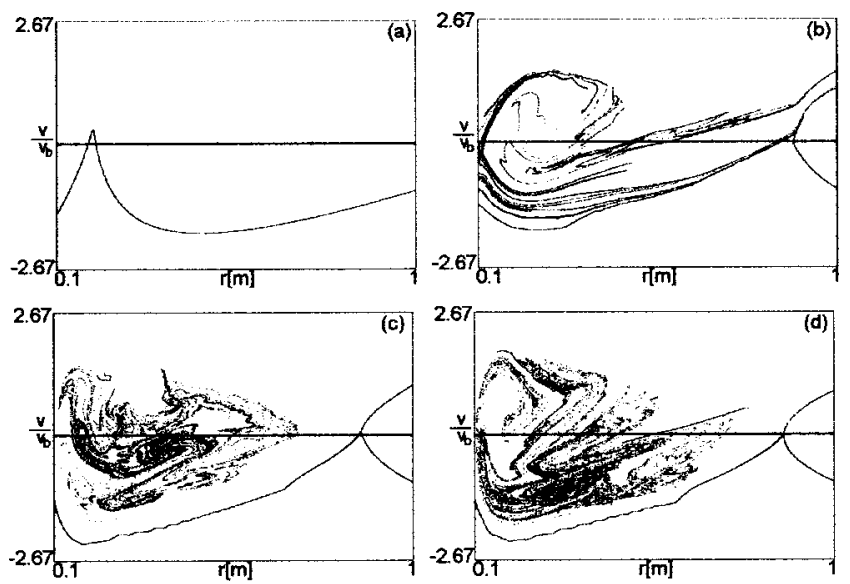

FIG. 15. Phase space, $v$ vs $r$, at different times, for $x_{i}=0.1$ and $\mu=3.8$. (a) $t=10 \tau$, (b) $t=14 \tau$, (c) $t=20 \tau$, and (d) $t=60 \tau$.

structure of the acceleration regions is almost identical. We may note, however, that the population of trapped particles at $60 \tau$ has decreased with respect to that at 10 or $20 \tau$. From observations of many similar runs we conclude that a typical energy gain in the jets is about 3 times the initial energy. The growth of the instability of the constant velocity states is related to an increase of electrostatic energy. This growth is interrupted by the transfer of energy to selected groups of electrons in the acceleration and deceleration processes mentioned above.

We now report the results for the convergent case $x_{i}$ $=0.1$. The four panels (a) $-(d)$ in Fig. 15 give phase space portraits at 10,14, 20, and 60 transit times, respectively. As in the previous experiment, the diode was started at a constant velocity state and perturbed by numerical noise. At $10 \tau$, in panel (a), we can see the first breakdown of the laminar flow, as some electrons are being reflected close to the collector. Four transit times later, in panel (b) we observe that a virtual cathode is present on the emitter side. On the absorber side a phase space vortex is being formed. The emitter is reabsorbing some freshly injected particles from the virtual cathode and also some reflected particles coming from the vortex region. On the collector side a considerable velocity spread is already apparent. At $20 \tau$, in panel (c), a potential well for electrons with a large population of trapped particles has developed on the collector side, and the virtual cathode near the emitter has been consolidated and is now a permanent feature of the system. Forty transit times later, the general pattern is still present and persists. A group of particles from the vortex region is being ejected towards the emitter. The population of trapped particles has increased from panel (b) to (d), so that the number of particles in the system oscillates but in average is larger than in the initial configuration. The fluctuation in the number of particles is due to the fact that the vortex region is losing particles to the collector and a fraction also to the emitter. Clearly, a group of particles can travel through the system without being trapped. These particles must be accelerated to large energies to overcome the electron potential well. The largest energy gain in panel (d) is about 5.6 times the initial energy. While in the divergent case the patterns seen in panels (b) to (d) in 
Fig. 14 are snapshots of similar phases of intermittent processes, panels (b) to (d) in Fig. 15 for the convergent case describe a continuous evolution of the same basic process.

From this and several other similar numerical experiments with convergent diodes we conclude that the instability growth saturates by two effects: the formation of a permanent virtual cathode which shortens the effective interelectrode distance, while at the same time limits the current because of the reflected electrons; simultaneously, a fraction of electrons does not contribute to the net electric current because a trapped particle population with zero average current is formed.

The difference in the nonlinear dynamics of convergent and divergent diodes can be summarized as follows. In divergent diodes, the nonlinear evolution of the instability leads to a number of electrons slightly smaller than in the initial state, the presence of the virtual cathode is intermittent, and the instability is reduced by transient periods of acceleration of groups of electrons. In convergent diodes, however, the saturation of the instability allows for an increase in the number of particles in the system with respect to the initial value, leading to the trapping of particles and the establishment of a permanent virtual cathode. We suggest that the different behavior during the unsteady regimes can be traced back to the fact that the electrons find a decreasing local plasma frequency as they approach the collector in divergent flows, and the Maxwell electric tension (see Sec. III E) does a positive work on expanding material volumes, while the opposite is true in convergent diodes.

\section{CONCLUSIONS}

In this paper we have shown the importance of steady state electrostatic structures in the nonlinear evolution of spherical diode flows. The numerical simulations have shown systematically, both in divergent and convergent configurations, that often the diode evolution can relax toward those states and remain permanently at them, starting from perturbations of constant velocity states. These nonuniform velocity states have a laminar electron motion and the electric current output of the diode is not modified.

We have given a theoretical description of the existence and stability diagram of the electrostatic states in the $E_{0}, \mu$ plane for two reciprocal values of $x_{i}, 10$ and 0.1 , based on the fluid model. The simulations have confirmed all the properties pointed out by the diagrams which we have investigated with several numerical experiments.

The theory of spherical diodes shows a duality in certain properties of the electrostatic steady states and of the spectrum of eigenvalues of small perturbations of these states, when diodes with $x_{i}$ and $1 / x_{i}$ ratios are examined for the same value of $\mu$. For instance, the electrostatic potential profile of a steady state for an $x_{i}$ diode is related to the potential profile of an $1 / x_{i}$ diode by a simple rescaling. The characteristic frequencies of the oscillations about these states (measured in transit time units) are the same. So that, for instance, the growth rates for the linear instability are equal for both dual configurations. The numerical simulations have fully confirmed the existence of this duality for reciprocal $x_{i}$ and
$1 / x_{i}$ diodes. This feature is a striking characteristic of the spherical diodes and does not hold in cylindrical Pierce configurations.

While there are some properties common to dual convergent and divergent diodes, when the laminarity of the flow is disrupted by the instability of the uniform constant velocity states (electron overcrossing and multivalued velocity) completely different dynamics develop for divergent and convergent diodes (even when pairs with reciprocal ratios, $x_{i}, 1 / x_{i}$ are considered). In both cases lack of order in phase space, $v$ vs $r$, arises, but with different physical features. In the divergent case the instability growth is saturated by intermittent acceleration processes of electron jets, with patterns that repeat quasi-periodically. In convergent diodes, instead, the instability is limited by the formation of a permanent virtual cathode and the growth of a large population of trapped particles.

The numerical experiments allowed us to discover a new property of divergent flows, which marks a difference in the time-varying operation of divergent and convergent diodes. This is the possibility of a spontaneous recovery of a laminar state (a stable electrostatic structure) after the formation of virtual cathode and the temporary disruption of laminarity (see, Secs. V A and V B, interval $2.4<\mu<3.4$ ). The divergent diode after leaving the unstable initial state shows an increase of disorder in the electron phase space, but since it is a driven open system it is capable of decreasing the disorder after some time. As far as we know, it is the first time that this effect has been reported in the context of the Pierce instability literature.

Another interesting result of the simulations was that permanent oscillatory electrostatic structures with welldefined frequencies can be formed, both in convergent and divergent diodes, for special values of the control parameters, $x_{i}, \mu$. These are observed in coincidence with the Hopf bifurcations predicted by the theory (Sec. IIIC).

Properties of the dispersion relation for perturbations of the initial constant velocity states have been studied for $x_{i}$ $=10$. These results also hold for the reciprocal diode with $x_{i}=0.1$. For spherical diodes the threshold of the first unstable mode appears for $\mu>1 / 2$. For $\mu<1 / 2$ all diodes are stable independent of the value of $x_{i}$, and therefore for any inter-electrode distance. This stability property was verified with sample numerical experiments. Such property does not hold for cylindrical or planar diodes.

The stability of some electrostatic structures for $\mu$ $>1 / 2$, and the possibility to access them which was confirmed by the simulations, means that, in principle, the current (or density) of the diodes can be increased to values substantially larger than the limit set by the first threshold $\mu_{1}$, by keeping a steady laminar flow through the system and a constant current output. This result has potential value for applications, in particular for devices with simultaneous ion beam injections for current neutralization. This property leads also to the interesting question about the influence of the ion dynamics on long time scales, which we hope to address in a future paper. 


\section{ACKNOWLEDGMENTS}

M.V.A. is grateful to the Brazilian Agencies: CNPq (Conselho Nacional de Desenvolvimento Científico e Tecnológico), and FUNCATE (Fundação de Ciência, Aplicações e Tecnologia Espaciais). G.G. and F.T.G. are members of the Argentine National Research Council (CONICET). Their work was supported by a CONICET Grant No. PIP 4536/96, and grant TX32 from UBACYT.

${ }^{1}$ S. Kuhn, Proceedings of the 1987 International Conference on Plasma Physics, Kiev, edited by A. G. Sitenko (World Scientific, Singapore, 1987), Vol. 2, p. 954.

${ }^{2}$ H. Kolinsky, F. Greiner, and T. Klinger, J. Phys. D: Appl. Phys. 30, 2979 (1997).

${ }^{3}$ H. Matsumoto, H. Yokoyama, and D. Summers, Phys. Plasmas 3, 177 (1996).

${ }^{4}$ H. Kolinsky and H. Schamel, J. Plasma Phys. 57, 403 (1997).

${ }^{5}$ T. L. Crystal and S. Kuhn, Phys. Fluids 28, 2116 (1985).

${ }^{6}$ B. B. Godfrey, Phys. Fluids 30, 1553 (1987).

${ }^{7}$ W. S. Lawson, Phys. Fluids B 1, 1493 (1989).

${ }^{8}$ N. Krahnstöver, T. Klinger, F. Greiner, and A. Piel, Phys. Lett. A 239, 103 (1998).

${ }^{9}$ H. Friedel, R. Grauer, K. H. Spatschek, Phys. Plasmas 5, 3187 (1998).

${ }^{10}$ Yu V. Vorob'ev, and V. A. Zhukov, Sov. Phys. Tech. Phys. 32, 81 (1987).

${ }^{11}$ A. D. Dunn, Models of Particles and Moving Media (Academic, New York, 1971)
${ }^{12}$ A. G. Nikonov, I. M. Roife, Y. M. Svel'ev, and V. I. Enger'ko, Sov. Phys. Tech. Phys. 32, 50 (1987).

${ }^{13}$ G. H. Miley, in Current Topics in Astrophysical and Fusion Plasmas, edited by M. Heyn and W. Kernbichler (DBV-Verlag, Graz, Austria, 1992), p. 103.

${ }^{14}$ J. W. Poukey, J. P. Quintenz, and C. L. Olson, Appl. Phys. Lett. 38, 20 (1981).

${ }^{15}$ J. W. Poukey, J. P. Quintenz, and C. L. Olson, J. Appl. Phys. 52, 3016 (1981)

${ }^{16}$ G. Gnavi and F. T. Gratton, Phys. Plasmas 1, 3676 (1994).

${ }^{17}$ M. V. Alves, F. T. Gratton, G. Gnavi, and C. H. Moreno, Phys. Plasmas 4, 3049 (1997).

${ }^{18}$ G. Gnavi, S. Ferro, F. T. Gratton, and M. V. Alves, Stability and bifurcations of electrostatic structures in spherical Pierce diodes, ECA 22C 619622 (1998), 1998 International Congress on Plasma Physics and 25th Conference on Controlled Fusion and Plasma Physics, Praha, 29 June-3 July, edited by P. Pavlo (European Physical Society, Petit-Lancy, 1999).

${ }^{19}$ C. Canuto, M. Y. Hussaini, A. Quarteroni, and T. A. Zang, Spectral Methods in Fluid Dynamics (Springer-Verlag, New York, 1988).

${ }^{20}$ C. K. Birdsall and A. B. Langdon, Plasma Physics via Computer Simulations (McGraw-Hill, New York, 1985).

${ }^{21}$ J. P. Verboncoeur, M. V. Alves, V. Vahedi, and C. K. Birdsall, J. Comput. Phys. 104, 321 (1993).

${ }^{22}$ M. V. Alves, M. A. Lieberman, V. Vahedi, and C. K. Birdsall, J. Appl. Phys. 69, 3823 (1991).

${ }^{23}$ C. K. Birdsall, IEEE Trans. Plasma Sci. 19, 65 (1991). 